\title{
Titanium alloy design and casting process development using an Integrated Computational Materials Engineering (ICME) approach
}

\author{
Zhi Liang ${ }^{1,2}$, Jiashi Miao ${ }^{1}$, Anil K. Sachdev ${ }^{3}$, James C. Williams ${ }^{1,5}$, and Alan A. Luo ${ }^{1,4}$ \\ ${ }^{1}$ Department of Materials Science \& Engineering, The Ohio State University, Columbus, \\ OH 43210, USA
}

2 Now at National Institute of Standards \& Technology, Gaithersburg, MD 20878, USA

3 Global Research and Development, General Motors, Warren, MI 48090, USA

${ }^{4}$ Department of Integrated Systems Engineering, The Ohio State University, Columbus, OH 43210, USA

${ }^{5}$ Department of Materials Science \& Engineering, University of North Texas, Denton, TX 76203, USA

\begin{abstract}
The application of titanium components is generally limited by their high raw material and manufacturing costs. In this paper, a lower cost cast titanium alloy based on the Ti-Al-Fe system has been designed using an ICME approach. The new alloy Ti-6Al-5Fe-0.05B-0.05C (all wt.\%) significantly reduces raw material cost and demonstrates improved castability compared with the baseline Ti-6Al-4V alloy. The fine primary and secondary $\alpha$ phase microstructure in the new alloy, due to Fe partitioning, provides exceptionally high strength (1023 MPa yield strength and $1136 \mathrm{MPa}$ ultimate tensile strength) and reasonable ductility (3.7\% elongation) for structural applications. On the manufacturing front, the high cost multi-step investment casting process currently used can now be replaced with a low-cost permanent mold casting process using steel molds and a novel ceramic coating. An experimental casting setup, including an induction skull melting (ISM) system, a gravity tiltpour system and a ceramic-coated H13 steel mold, has been used to produce near-net-shape permanent metallic mold castings with the new titanium alloy developed. Using this setup, and aided by casting process simulation, a prototype automotive connecting rod was cast
\end{abstract}


successfully. The $\mathrm{ZrO} 2$ ceramic coating applied to the $\mathrm{H} 13$ steel mold was proven effective in minimizing the metal-mold reactions.

\section{Introduction}

Titanium alloys are important engineering materials for high value lightweight products in structural applications due to their superior mechanical properties, corrosion resistance, weldability, and low density [1]. The relative high property-to-weight ratios of titanium alloys are ideal for critical and weight-sensitive applications, such as airframes and other aeronautics applications [2]. However, the application of titanium alloys for high volume automotive applications is still scarce due to high manufacturing and alloy cost [3]. Currently, Ti-6Al-4V (Ti-6-4) is the workhorse titanium alloy used in almost all titanium components, with $90 \%$ of casting products made from Ti-6-4. There is, therefore, a need for a lower cost alloy, especially one without vanadium, and a lower cost casting process instead of normally used investment casting.

Integrated Computational Materials Engineering (ICME) has gained increasing interest in designing new alloys and process development. Specifically, CALculation of PHAse Diagram (CALPHAD) modeling within an ICME framework is essential to provide information on phase stability and transformations for alloy design [4]. In this investigation, the design of a new Ti-Al-Fe-based alloy was guided by CALPHAD modeling. Process development, on the other hand, was aided by casting process simulation and optimization using commercial software.

As mentioned earlier, the Ti-Al-Fe system was selected to replace the Ti-Al-V system to reduce the raw material cost by substituting $\mathrm{V}$ with $\mathrm{Fe}$. In addition to its low cost, Ti-Al-Fe can potentially improve castability compared with the Ti-Al-V system by significantly decreasing the liquidus temperature and enlarging the liquidus-solidus gap as predicted by CALPHAD (Figure 1). The Ti-Al-Fe alloy system was explored in the 1980-90s for hot working processes, and a few compositions were developed [5-7]. However, no systematic investigations have been reported for this alloy system. In this investigation, a new cast titanium alloy was designed by a combined CALPHAD and experimental approach. 


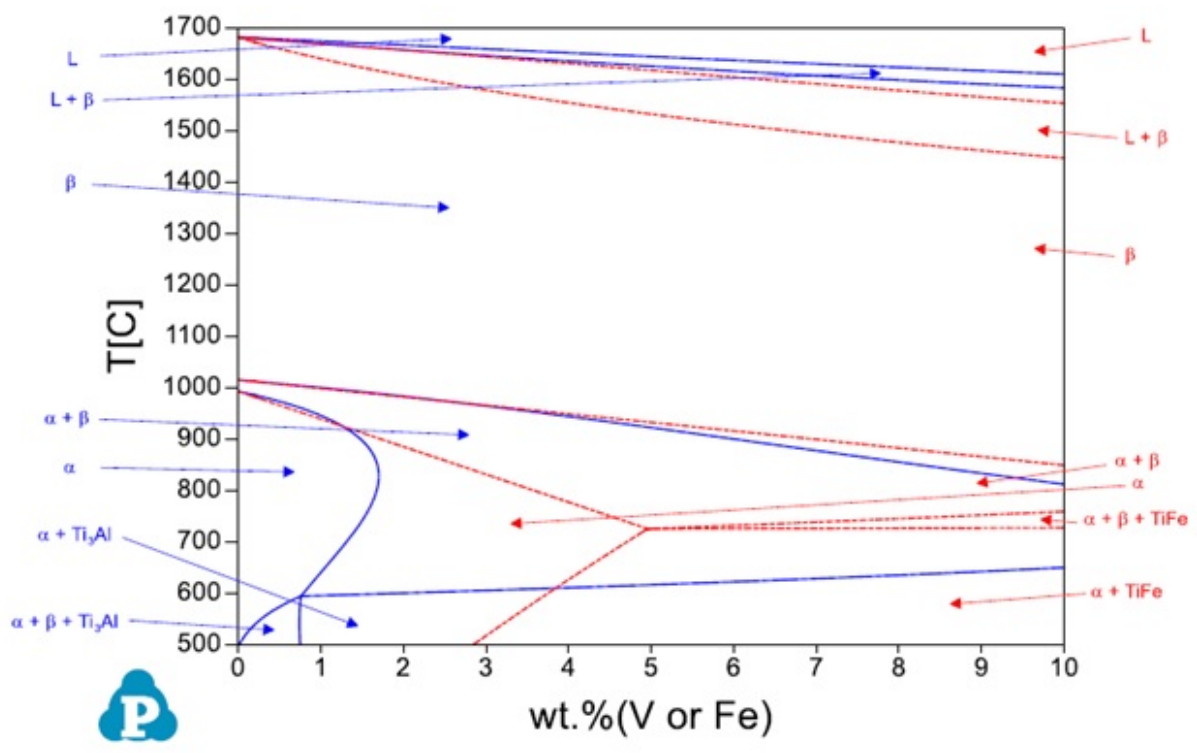

Figure 1. Comparison of calculated isopleths of Ti-6Al-xV (blue) and Ti-6Al-xFe (red), using PANDAT software and PanTi_2017 database [8].

Currently there are two major casting processes for titanium alloys, i.e., graphite mold casting [9] and investment casting [10], and both can produce components for low-volume production. However, both techniques use expendable molds and are labor-intensive. They are thus not suitable for high-volume production such as the automotive industry. On the other hand, permanent mold casting, the common high-volume casting technique, is difficult for titanium due to contamination and mold damage issues. Therefore, in this investigation, the permanent metallic mold casting was improved to make it a viable process for producing titanium components.

\section{Experiments and Simulation}

\subsection{CALPHAD-based Alloy Design}

The baseline alloy in this investigation is Ti-6-4 due to its well-documented properties and microstructure and, as such, was used for comparison. The Al content was controlled at 6 wt.\%, the same as the baseline alloy, to provide $\alpha$ solid solution strengthening while avoiding precipitation of the $\alpha 2$ phase and ordering of the $\beta$ phase, both of which cause loss of ductility. The Fe content was determined by the CALPHAD isopleth results as shown in Figure 1. An Fe content of $5 \mathrm{wt} . \%$ maximized the $\alpha+\beta$ two phase temperature range and was chosen to allow $\alpha$ precipitation while avoiding possible formation of brittle TiFe 
intermetallics. In addition, trace levels of $\mathrm{B}$ and $\mathrm{C}$ were included in this new alloy for grain refinement [11-13] for a final alloy composition of Ti-6Al-5Fe-0.05B-0.05C (T65-005BC, wt.\%). The relevant detailed literature was discussed in an earlier publication by the authors [14].

\subsection{Casting Setup and Mold Design}

The prototype casting setup in this investigation included induction skull melting (ISM) and permanent mold casting. The melting and casting concept, proposed by the authors, was designed and built by PVT Inc. (Rancocas, NJ), an InductoTherm Group Company [15], shown in Figure 2. The ISM system has a 500-gram melt capacity for titanium alloys, a mold-heating unit, and a tilt-pour system. The mold design is a modification based on an automotive engine connecting rod provided by General Motors. To achieve better cavity filling, the connecting rod geometry was split into two parts, with the I-beam and the smallring features removed to simplify molten metal flow as shown in Figure 3.
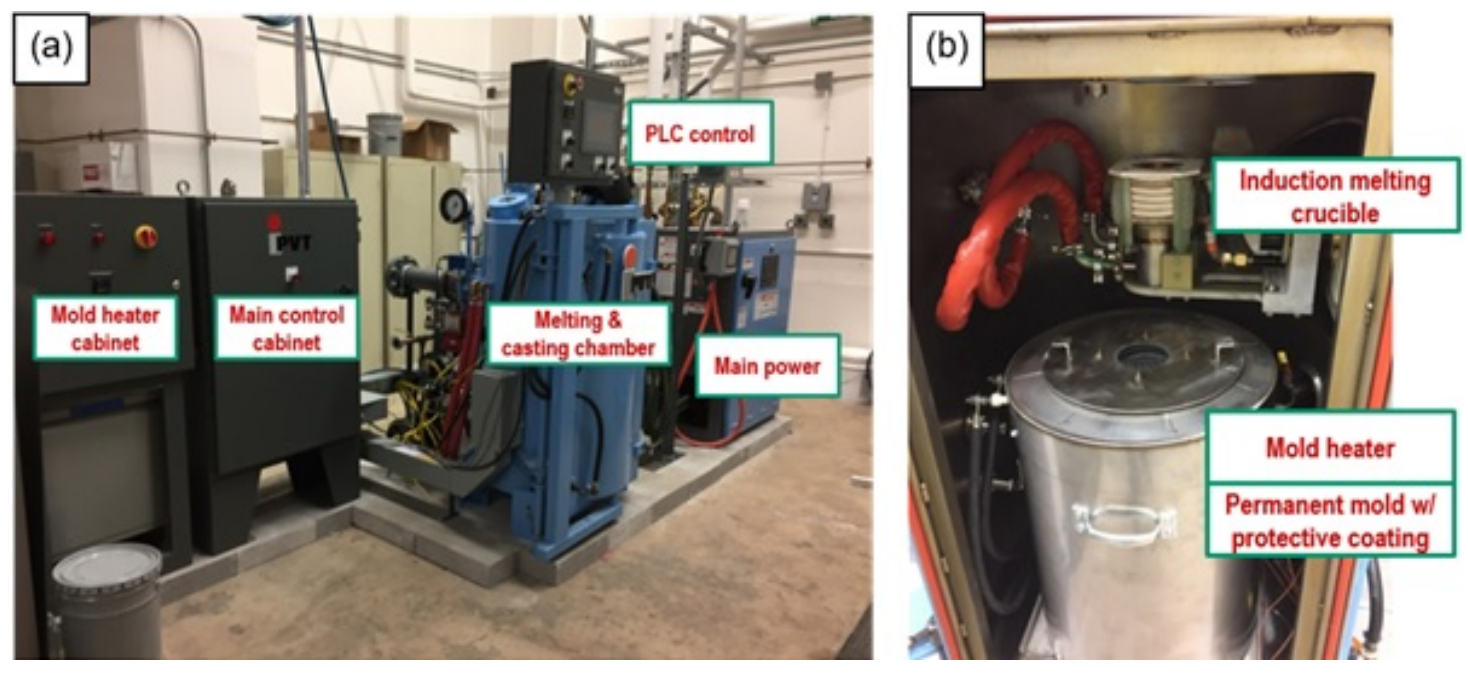

Figure 2. Titanium melting and casting system at OSU: (a) overall view, including all control, power cabinets, and vacuum chamber; and (b) in-chamber view, including ISM crucible and mold heater. The ISM crucible can be tilted with a mechanical lever outside of chamber for tilt-pouring. 

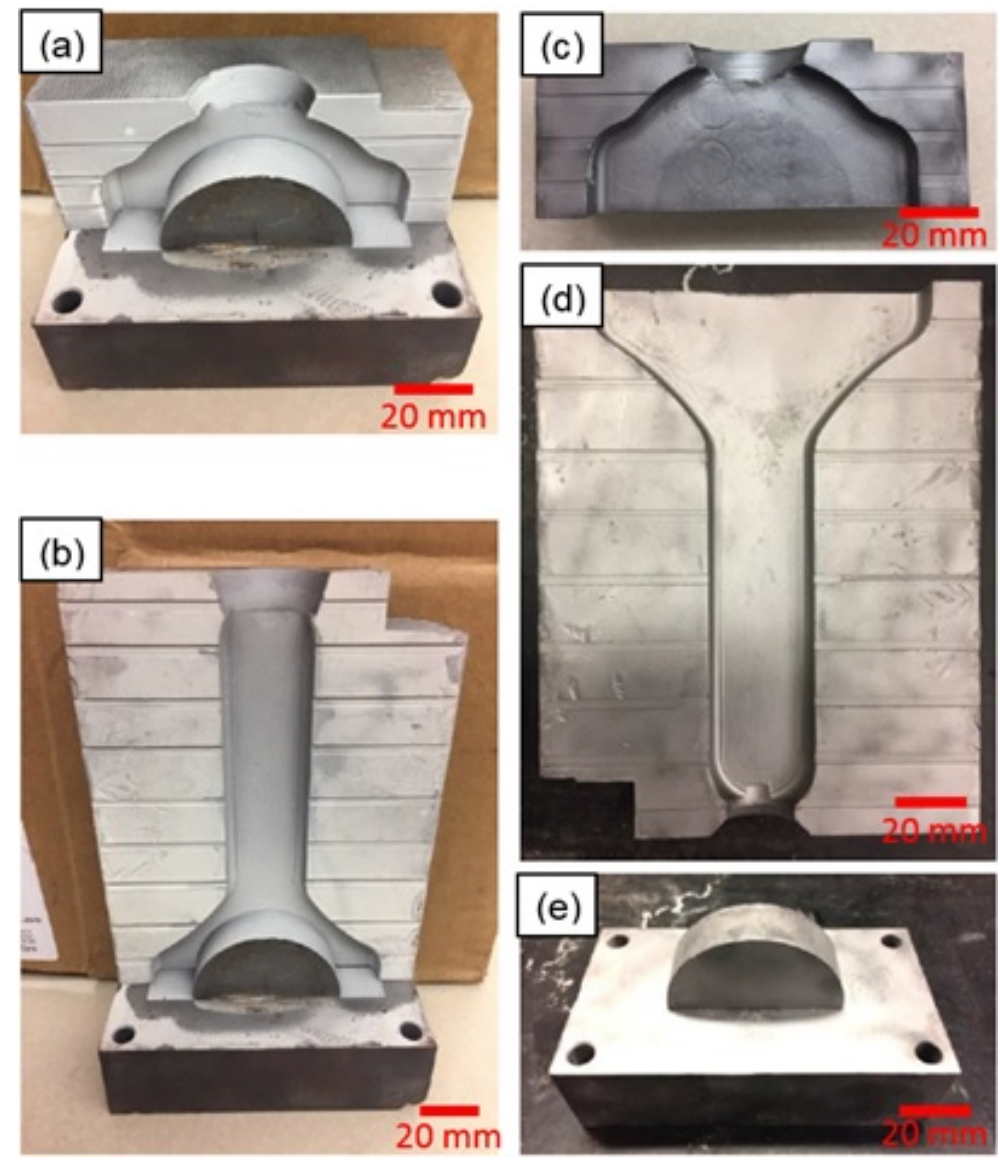

Figure 3. Pre-casting mold with $\mathrm{ZrO} 2$ coating of (a) top half and (b) bottom half; and postcasting mold pictures of (c) top half, (d) bottom half, and (e) core after 10 casting cycles.

\subsection{Alloy Melting and Casting}

Five hundred grams of an experimental alloy with the nominal composition of Ti-6Al-5Fe$0.05 \mathrm{~B}-0.05 \mathrm{C}$ (designated as $\mathrm{T} 65-0.05 \mathrm{BC}$, wt $\%$ ) was prepared using low oxygen, commercially pure Grade 2 titanium (courtesy of ATI, Albany OR), commercial pure aluminum (99.9\%, Alfa Aesar, Haverhill, MA), commercial pure iron (99.9\%, Alfa Aesar), TiB2 (99.5\%, Alfa Aesar), and carbon slug (99.5\%, Alfa Aesar). During melting and casting, the chamber was evacuated and backfilled with argon to 80 torr (about $0.1 \mathrm{~atm}$ ) to minimize air in the chamber and evaporation of aluminum from the melt. Prior to casting, the alloy was re-melted three times to ensure homogeneity. An extra ingot was made to examine the water-cooled alloy microstructure. The oxygen analysis, tensile test and characterization procedures also were described by authors in an earlier publication [14].

The charge materials were first pre-heated in the crucible to avoid large temperature 
gradients during melting, preventing large solid chunks from sinking to the bottom of the melt pool. During pre-heating, the induction power output was set at $10 \mathrm{~kW}(20 \%$ of maximum power) for 15-20 minutes. From the observation port of the chamber, the preheating should be considered complete once the radiation of the charge materials was evenly distributed. The power was then gradually increased to the maximum of $50 \mathrm{~kW}$. The induction frequency was $10000 \pm 100 \mathrm{~Hz}$. As soon as the charge materials reached the molten state and the melt pool stirring from the induction field was stable, it was kept at maximum power output for 10-15 minutes to ensure that the charge materials were completely melted and well mixed. Following this, the $\mathrm{ZrO} 2$-coated pre-heated $\mathrm{H} 13$ steel mold was filled by tilt-pouring the molten metal. In this investigation, the mold was pre-heated to $700^{\circ} \mathrm{C}$ to enhance molten metal fluidity. Before tilt-pouring, the induction power was turned down to $25 \mathrm{~kW}$ (50\% of the maximum power) to prevent the molten metal from splashing while retaining the superheat. After filling, the mold-heating and induction power were turned off to let the mold and crucible cool down inside the chamber for about 30 minutes, following which the vacuum was released for removing the mold and the casting. Before the next casting, the mold was cleaned with grit blasting and re-coated with $\mathrm{ZrO} 2$. There were in total 10 re-use cycles of melting and casting in this study.

\subsection{Casting Simulation}

Casting simulation of mold-filling and solidification was conducted to optimize the casting design and reduce the possibility of casting defects. In this work, EKK Capcast [16] commercial casting simulation code was used, and the following simulation results were used for process optimization: fill velocity, solidification sequence, and flow simulation. These results revealed potential inhomogeneity and hot spots during filling and solidification. Several input parameters were calculated using CALPHAD and applied in the simulation, including liquidus $\left(1608^{\circ} \mathrm{C}\right)$ and solidus $\left(1443^{\circ} \mathrm{C}\right)$ temperatures of the titanium alloy. The density of the alloy was measured as $4.45 \mathrm{~g} / \mathrm{cm} 3$. The thermal conductivity, 6.7 $\mathrm{W} / \mathrm{m}-\mathrm{K}$, heat capacity, $0.5263 \mathrm{~J} / \mathrm{g}-{ }^{\circ} \mathrm{C}[17]$, viscosity, $4.76 \mathrm{mPa} \cdot \mathrm{s}$, surface tension, $1.52 \mathrm{~N} \cdot \mathrm{m}-$ 1 [18], latent heat of fusion, $290 \mathrm{~kJ} / \mathrm{kg}$ [19], reported for the Ti-6Al-4V alloy, were used in this simulation study.

Representative casting simulation results are shown in Figure 4. As observed in the upper casting, the differences in filling time and post-filling temperature distribution are not severe, indicating that shrinkage defects were less likely to occur. On the other hand, in the lower casting, the difference in filling time and post-filling temperature distribution are relatively higher, indicating potential shrinkage defects due to sequential solidification. It can also be observed that the long and steep filling path causes significant turbulence during filling and splashing of the molten metal on the mold surface, likely causing early solidification on to the mold surface. This disturbs the subsequent metal flow, creating surface defects. Due to the limitation of the current experimental setup, multiple experimental variables were simplified in the simulation. Therefore, the simulation results are used only as general guidance to minimize potential casting defects. 


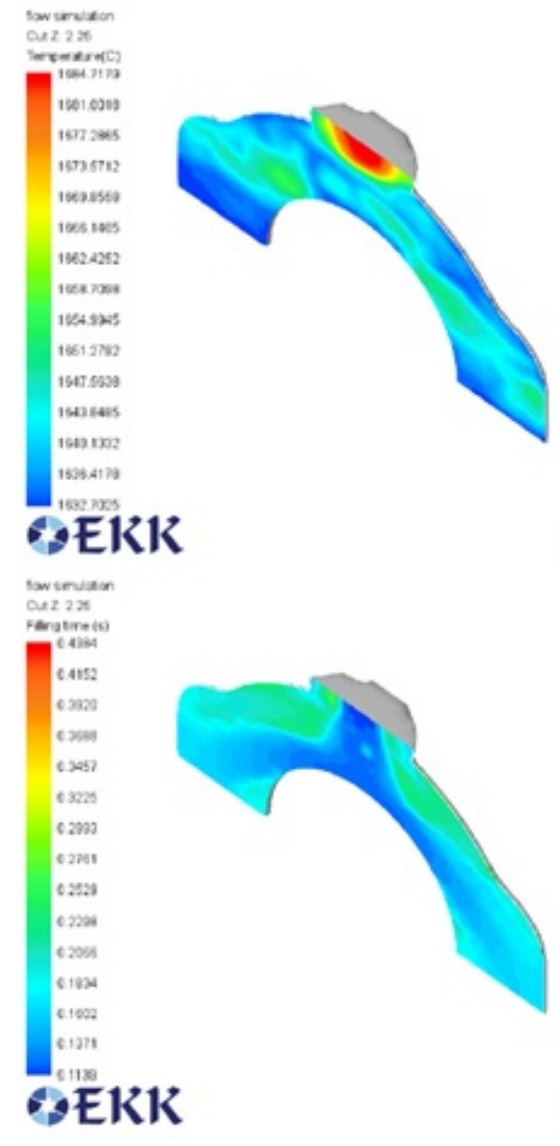

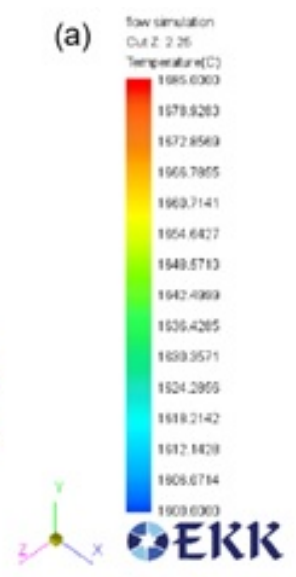

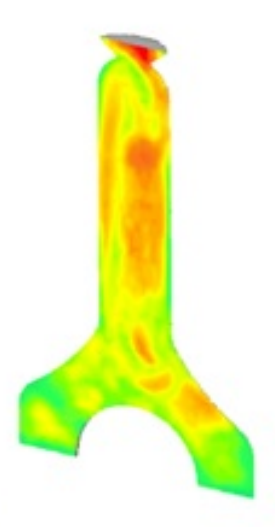

(b)

(c)

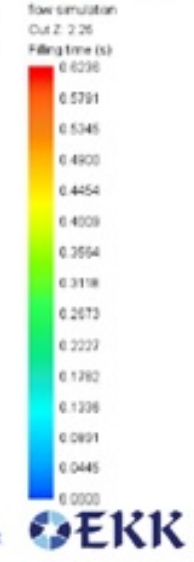

(d)

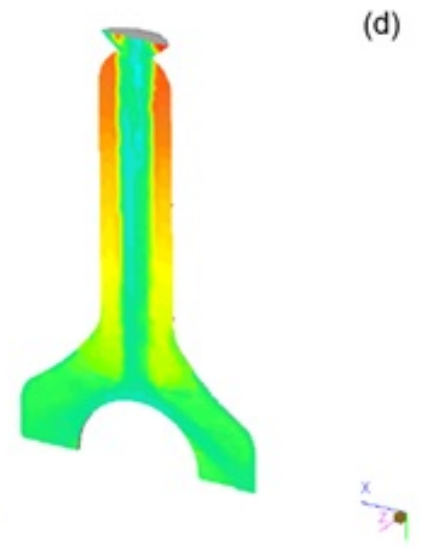

Figure 4. Cavity fill and solidification simulation results of connecting rod casting: $(a, b)$ final temperature after solidification; and (c, d) filling time of (a, c) upper half and $(b, d)$ lower half.

\section{Results and Discussion}

\subsection{Microstructure Characterization and Property Evaluation}

The microstructure of a water-cooled ingot of the new alloy is shown in Figure 5(a-d). As is observed, fine primary and secondary $\alpha$ phases are present in the microstructure, but no continuous grain boundary $\alpha$ phase is present. The stress-strain curve for this alloy is shown in Figure 5(e) and the resulting properties are compared with several other commercial ascast titanium alloys in Table 1. The new alloy had comparable strength to Ti64, but much lower ductility, probably due to the high $\alpha$ phase fraction. The details of this new alloy were included in an earlier publication by the authors and not repeated in this article. 

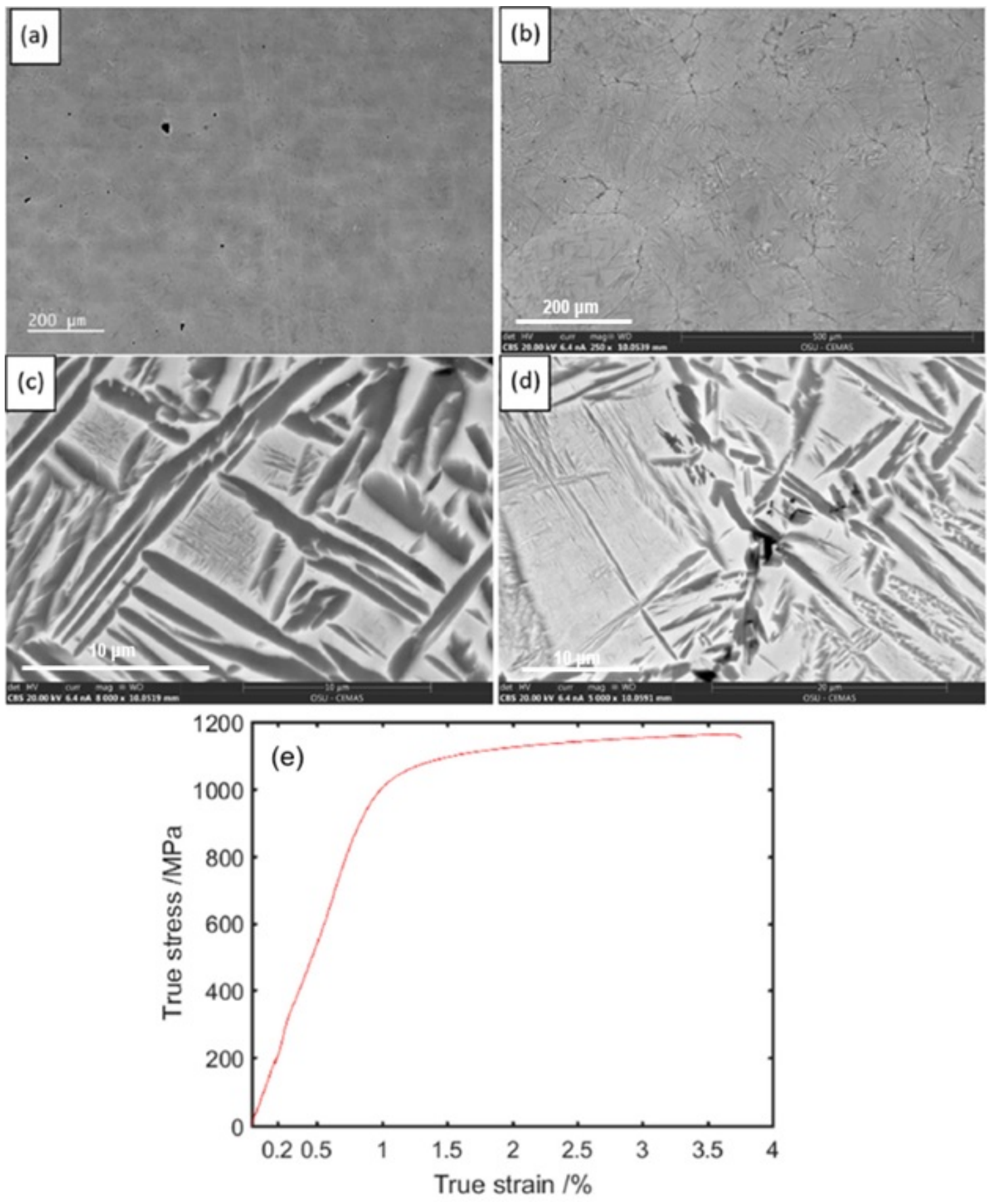
Figure 5. (a-d) SEM backscattered images of as-cast Ti-6Al-5Fe-0.05B-0.05C; and (e) ASTM E8 tensile stress vs. strain curve [14].

Table 1 Tensile properties of as-cast Ti-6Al-5Fe-0.05B- $0.05 \mathrm{C}$ compared with as-cast Ti-6Al4V, Ti-6Al-4V ELI [20], and Ti-5Al-2.5Fe [17, 21].

\begin{tabular}{|l|l|l|l|}
\hline As-cast Material & $\begin{array}{l}\text { Yield Strength } \\
(\mathrm{MPa})\end{array}$ & $\begin{array}{l}\text { Ultimate Tensile Strength } \\
(\mathrm{MPa})\end{array}$ & $\begin{array}{l}\text { Elongation } \\
(\%)\end{array}$ \\
\hline $\begin{array}{l}\text { Ti-6A1-5Fe-0.05B- } \\
0.05 \mathrm{C}\end{array}$ & $1177.67 \pm 47.61$ & $1284.67 \pm 31.89$ & $1.38 \pm 0.28$ \\
\hline Ti-6Al-4V & 895 & 1000 & 8 \\
\hline Ti-6Al-4V ELI & 825 & 896 & 10.5 \\
\hline Ti-5Al-2.5Fe & 820 & 900 & 6.0 \\
\hline
\end{tabular}

\subsection{Prototype Casting and Mold Evaluation}

A rough polished prototype casting with least surface defects is shown in Figure 6. The H13 steel mold, coated with about $0.5 \mathrm{~mm}$ thick $\mathrm{ZrO} 2$ was successfully filled by gravity-pour. Although no large unfilled or shrinkage areas are observed, as shown in Figure 6, the casting defects on the component surface are visible, including gas porosity and surface roughness. These defects are due to the experimental limitations in this investigation.

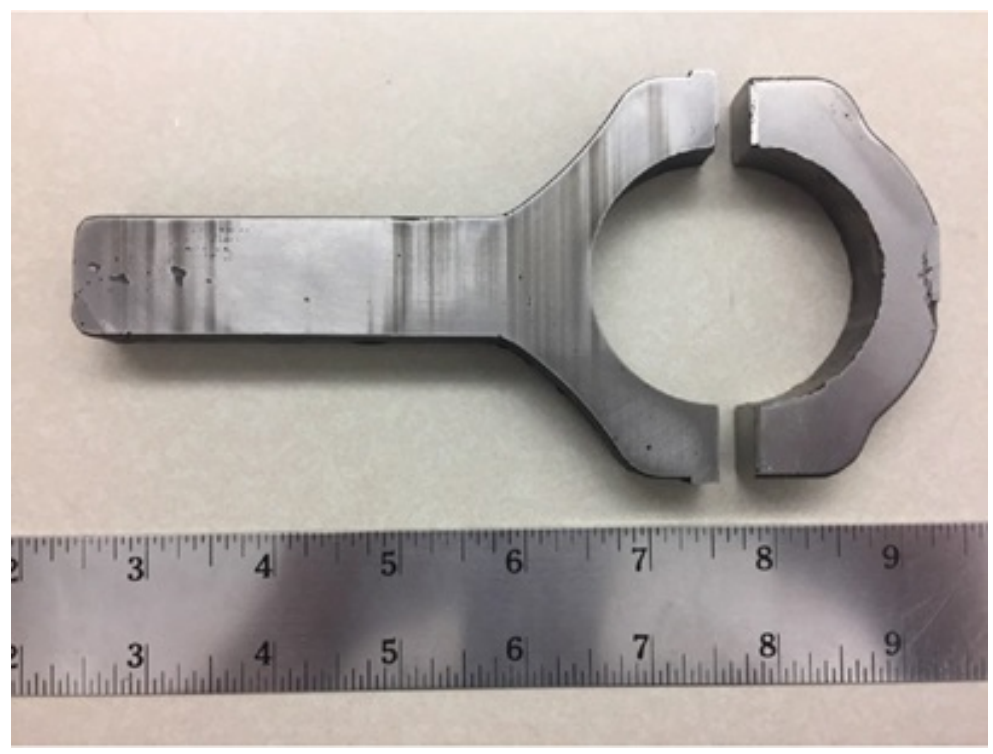


Figure 6. Rough polished prototype casting Ti-6Al-5Fe-0.05B-0.05C connecting rod. Reference scale ruler in inches.

The pre-casting and post-casting mold pictures are shown in Figure 3. As can be seen, the mold is not damaged from contact with the melt, but the coating has peeled off from the mold surface during casting removal. This was a result of poor coating adhesion due to airspraying used in this study. However, as shown in Figure 3, the mold is protected against molten titanium. There was some $\mathrm{ZrO} 2$ coating adhered to the casting, but it was easily removed with grit blasting.

\subsection{Recommendations for Further Process Improvement}

Based on the outcomes of this investigation, the following improvements should be considered in scaling up this process:

1) ISM melting system: Scale up of the permanent mold casting process should consider higher capacity ISM to cast multiple parts in each cycle to increase productivity.

2) Mold for multiple parts and centrifugal filling-assisted system: One of the mature methods in making small castings is the centrifugal casting setup, which will improve the filling performance for high-volume production. Centrifugal casting is a mature technique and has already been applied to the investment casting of titanium alloys. For example, Koike et al [22] applied on Ti-5Cu and CP-Ti, and Ling et al [23] were successfully is using this technique for investment casting of Ti-6Al-4V alloy.

3) Mold cooling system: No mold cooling system was used in our experimental setup due to experimental limitations. To achieve finer microstructure in the castings, the mold should be cooled faster than this setup allows preferably with a deionized-water/oil cooling line through the mold. The mold cooling should start as soon as the filling is complete.

4) Computer-controlled tilt-pour system: This system would allow precise control of the tilt-pour in casting operations, enhance the repeatability of the process, and improve the final component quality.

5) Thermal/plasma-spraying of the ceramic coating: Instead of air-spraying the coating as in this investigation due to experimental limitations, thermal/plasma-spraying can provide increased coating adhesion with the mold, allowing a greater number of casting cycles per coating to improve coating life and efficiency. 


\section{Conclusions}

This work successfully developed a new low-cost cast titanium alloy and established a labscale titanium casting setup which proves the concept of permanent metallic mold casting for titanium alloys. The major conclusions are as follows:

1) A new cost-effective cast titanium alloy Ti-6Al-5Fe-0.05B-0.05C was developed by substituting $\mathrm{Fe}$ for $\mathrm{V}$ to significantly reduce raw material cost and provide good castability. This new alloy has very high strength due to the primary $\alpha$ lamellae and ultra-fine secondary $\alpha$ precipitates. Its strength-ductility can be tailored by heat treatment for various applications.

2) Permanent mold casting experiments of titanium alloys using a $\mathrm{H} 13$ steel mold and $\mathrm{ZrO} 2$ ceramic coating showed minimal reaction between the metallic mold and molten titanium and successfully prevented casting-mold reactions in permanent mold casting of titanium alloys. However, coating application processes that demonstrate better adhesion to the mold should be developed.

3) Aided by casting process simulation, a prototype connecting rod was successfully cast without significant casting defects (e.g. shrinkage cavity, cold shuts, etc.), but still showed minor surface defects (e.g. turbulence-induced gaps and gas porosities, etc.).

4) The lab-scale casting setup, including an induction skull melting (ISM) system, a gravity tilt-pour system, and a ceramic-coated H13 steel mold, has been used to successfully produce near-net-shape permanent metallic mold titanium castings.

5) This lab-scale demonstration shows promising results and should be developed further.

\section{Acknowledgement}

This investigation is sponsored by the U.S. Department of Energy under award DEEE0006858. This report was prepared as an account of work sponsored by an agency of the United States Government. Neither the United States Government nor any agency thereof, nor any of their employees, makes any warranty, express or implied, or assumes any legal liability or responsibility for the accuracy, completeness, or usefulness of any information, apparatus, product, or process disclosed, or represents that its use would not infringe privately owned rights. Reference herein to any specific commercial product, process, or service by trade name, trademark, manufacturer, or otherwise does not necessarily constitute 
or imply its endorsement, recommendation, or favoring by the United States Government or any agency thereof. The views and opinions of authors expressed herein do not necessarily state or reflect those of the United States Government or any agency thereof.

\section{References}

[1] Lütjering, G. and J. C. Williams, Titanium, Springer Berlin Heidelberg, 2007

[2] Campbell, F. C., Lightweight Materials: Understanding the Basics, ASM International, 2012

[3] Eylon, D. and F. H. Froes, JOM, 6 (1984) 36-41

[4] Luo, A. A., Calphad, (2015) 6-22

[5] Borowy, K. H. and K. H. Kramer. Titanium Science and Technology, Deutsche Gesellschaft für Metallkunde E.V., (1985) 1381-1386

[6] Bania, P. J., A. J. Hutt, R. E. Adams and W. M. Parris. Titanium'92 Science and Technology, TMS, Warrendale, (1993) 2787-2794

[7] Fujii, H. and K. Takahashi, Nippon Steel Tech. Rep., 85 (2002) 113-117

[8] CompuTherm-LLC (2019). from http://www.computherm.com/

[9] O'Connor, S., Advanced Materials \& Processes, 1 (2008) 29-31

[10] Pattnaik, S., D. B. Karunakar and P. K. Jha, Journal of Materials Processing Technology, 11 (2012) 2332-2348

[11] Roy, S., S. Suwas, S. Tamirisakandala, D. B. Miracle and R. Srinivasan, Acta Materialia, 14 (2011) 5494-5510

[12] Sarkar, R., P. Ghosal, T. K. Nandy and K. K. Ray, Philosophical Magazine, 15 (2013) 1936-1957

[13] Banoth, R., R. Sarkar, A. Bhattacharjee, T. K. Nandy and G. V. S. Nageswara Rao, Materials \& Design, (2015) 50-63

[14] Liang, Z., J. Miao, T. Brown, A. K. Sachdev, J. C. Williams and A. A. Luo, Scripta Materialia, (2018) 124-128

[15] InductoTherm-Group (2019). from https://www.inductothermgroup.com/

[16] EKK, I. (2018). "EKKcapcast." Retrieved October 29th, 2018, from https://www.ekkinc.com

[17] Welsch, G., R. Boyer and E. W. Collings, Materials Properties Handbook: Titanium Alloys, ASM International, 1993

[18] Wunderlich Rainer, K., Journal. 27 (2008) 401

[19] Boivineau, M., C. Cagran, D. Doytier, V. Eyraud, M.-H. Nadal, B. Wilthan and G. Pottlacher, International Journal of Thermophysics, 2 (2006) 507-529

[20] Billinghurst Jr., E. E., NASA Tech. Pap., 3288 (1992) 9-10

[21] Murphy, W., J. Black and G. Hastings, Handbook of Biomaterial Properties, Springer New York, 2016

[22] Koike, M., A. Krysiak, K. S. Chan, L. Guo and T. Okabe, Journal of Materials Processing Technology, 4 (2011) 560-565

[23] Ling, Y., J. Zhou, H. Nan, L. Zhu and Y. Yin, Journal of Materials Processing Technology, (2018) 295-304 